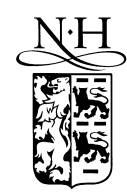

ELSEVIER

\title{
Planckian scattering of D-branes
}

\author{
Saurya Das a,b,1, Arundhati Dasgupta a,2, P. Ramadevi ${ }^{\text {c,3 }}$, Tapobrata Sarkar a,4 \\ ${ }^{\mathrm{a}}$ The Institute of Mathematical Sciences, CIT Campus, Chennai - 600113, India \\ b International Centre for Theoretical Physics, P.O. Box 586, 34100 Trieste, Italy \\ ${ }^{\mathrm{c}}$ Tata Institute of Fundamental Research, Homi Bhabha Road, Mumbai - 400 005, India \\ Received 18 February 1998 \\ Editor: L. Alvarez-Gaumé
}

\begin{abstract}
We consider the gravitational scattering of point particles in four dimensions, at Planckian centre of mass energy and low momentum transfer, or the eikonal approximation. The scattering amplitude can be exactly computed by modelling point particles by very generic metrics. A class of such metrics are black hole solutions obtained from dimensional reduction of $p$-brane solutions with one or more Ramond-Ramond charges in string theory. At weak string coupling, such black holes are replaced by a collection of wrapped D-branes. Thus, we investigate eikonal scattering at weak coupling by modelling the point particles by wrapped D-branes and show that the amplitudes exactly match the corresponding amplitude found at strong coupling. We extend the calculation for scattering of charged particles. (C) 1998 Published by Elsevier Science B.V. All rights reserved.
\end{abstract}

\section{Introduction}

It is expected that the quantum gravity effects would become important at energies compared to the Planck Scale. Since the gravitational coupling constant $G$ is not dimensionless, one can construct two independent dimensionless coupling constants, which for example in four space-time dimensions can be defined as $G_{\|} \equiv G_{4} s$ and $G_{\perp} \equiv G_{4} t$. Here $G_{4}$ is the four dimensional Newton's constant, and $s, t$ are the Mandelstam variables. Remarkably, it was shown in [1] that the full theory of quantum gravity can be split up into two independent theories with these

\footnotetext{
${ }^{1}$ E-mail: saurya@imsc.ernet.in.

${ }^{2}$ E-mail: dasgupta@imsc.ernet.in.

${ }^{3}$ E-mail: rama@theory.tifr.res.in.

${ }^{4}$ E-mail: sarkar@imsc.ernet.in.
}

coupling constants. Thus, a quantum gravitational regime for gravitational scattering can be envisaged when either $G_{\|} \approx 1$ or $G_{\perp} \approx 1$, or both. While the former signifies Planckian centre-of-mass energies, the latter implies Planckian momentum transfers. The first quantum gravitational scenario is easier to deal with because although $s$ is large, $t$ can be held fixed at a relatively small value such that $s / t \rightarrow \infty$. The impact parameter of scattering, in this case, is very large and the scattering is almost forward. This is the so called eikonal approximation where the exact two particle scattering amplitude can be computed. In practice, it is advantageous to view one of the particles as static (say $A$ ) and the other moving (say $B$ ) at almost luminal velocity past it with a large impact parameter. $A$ being static, can be suitably modelled by a metric, whose gravitational field $B$ is supposed to experience. Then one can solve the 
wave equation for $B$ in this given background and obtain the scattering amplitude. Of course, the reverse process is equally valid, when the 'shock-wave' space time produced by the $A$ is obtained by Lorentz boosting the metric and analysing the wave function of the slow particle in this shock-wave background. As expected, the two pictures yield the same result [2].

In the above picture, the point particles are usually modelled by Schwarzschild or ReissnerNordström metrics, depending on whether the particles are neutral or charged. Although this seems natural in the framework of general relativity, these specific choices are certainly not mandatory. We show here that the results can be extended for a large class of generic spherically symmetric metrics. As an example, a large class of metrics arise as solutions of low energy string theory and one could model the particles by these metrics as well. The black holes which carry the NS-NS charges have already been considered in Planckian scattering which lead to interesting consequences [3]. It is shown here that these black hole metrics do not fall into the class of metrics we consider here, except in the extremal limit. Recently a new class of black hole solutions of low energy effective action of superstring theory have been found, whose weak coupling (in terms of the string coupling $g$ ) description consists of certain configurations of solitonic string states or D-branes, wrapped on suitable compact manifolds. Several pieces of evidence have emerged supporting this identification, the most notable being the fact that the degeneracy associated with the D-brane configuration exactly reproduces the Bekenstein-Hawking entropy [4] and open string interactions on the D-brane reproduce the Hawking radiation spectrum of these black holes [5]. Thus, while the black hole description can be used at large coupling, the D-brane description is appropriate at small coupling [4-6]. D-brane scattering has been considered in [7-12], and in particular in [7-10], it has been shown scattering amplitudes of R-R charged $p$-branes agree with appropriate D-brane scattering in ten dimensions. There are black hole solutions with a singular horizon obtained by wrapping these R-R charged $p$ branes on compact spaces. The entropy for these black holes is zero, and hence the appropriate process to check for D-brane black hole correspondence is to look at scattering amplitudes. In this paper, we show that indeed the exact eikonal scattering amplitude can be computed for wrapped D-branes at weak coupling. Moreover, this amplitude agrees with that found in the black hole picture. The agreement persists when the particles carry $U(1)$ charges also.

In the following section, we calculate the eikonal scattering phase shift in the strong coupling regime by modelling the particles by a general spherically symmetric black hole metric in four space-time dimensions. We also consider the special cases of R-R and NS-NS charged black holes. In the next section, we calculate the corresponding phase shift at weak coupling using $\mathrm{D}$ - $p$-branes wrapped on tori. The D-brane result is found to be independent of the brane dimensions as long as they are completely wrapped on the internal tori. Significantly, the scattering phase shift is dominated by graviton exchanges at ultrarelativistic velocities, as anticipated earlier in the calculations of [2]. Finally, we extend the calculations to include particles carrying electric charges, where too the results continue to agree.

\section{Eikonal scattering at large string coupling}

We will assume that the slow target particle gives rise to the following most general spherically symmetric metric in four dimensions:

$d s^{2}=-\lambda^{2} d t^{2}+\frac{1}{\lambda^{2}} d r^{2}+R r^{2} d \Omega^{2}$,

where $\lambda$ and $R$ are functions of the radial coordinate only.

In general let the ultrarelativistic particle of charge $e$ be minimally coupled to the $U(1)$ gauge field $A_{\mu}$ produced by the static particle. Then its wave function $\Phi$ satisfies the covariant Klein-Gordon equation

$$
\begin{aligned}
& \frac{1}{\sqrt{-} g} D_{\mu}\left(\sqrt{-g} g^{\mu \nu} D_{\nu} \Phi\right)+m^{2} \Phi=0, \\
& D_{\mu}=\partial_{\mu}-i e A_{\mu},
\end{aligned}
$$

where $m$ and $E$ are the mass and energy of the test particle. In the spirit of [2] we will assume that $M, m \ll M_{\mathrm{pl}}$, where $M_{\mathrm{pl}}$ is the Planck mass. In the centre of mass frame of the particles, their energies are proportional to $\sqrt{s}$ and for the particles to have relativistic velocities at this energy $M, m \ll \sqrt{s}$. Since 
in our problem $\sqrt{s} \sim M_{\mathrm{pl}}$ we get the above condition on the mass. Thus we will ignore all terms quadratic in $M, m$ in subsequent discussions. We substitute a solution of the form

$\Phi(\boldsymbol{r}, t)=\frac{\Phi^{\prime}}{r} e^{i E t} Y_{l m}(\theta, \phi)$,

and linearize the metric components at large distances as

$\lambda^{2}=1-\frac{2 G M}{r}, \quad R=1+\mathscr{O}(1 / r)$.

Here $M$ is the ADM mass of the black hole metric. Retaining terms upto order $1 / r^{2}$, the radial equation of the $\bar{l}^{\text {th }}$ partial wave is (for large $\bar{l}$ )

$$
\begin{aligned}
& \frac{d^{2} \Phi^{\prime}}{d r^{2}}-\left[\frac{\bar{l}(\bar{l}+1)}{r^{2}}-E^{2}-\frac{4 G_{4} M E^{2}}{r}+2 e E A_{0}\right] \Phi^{\prime} \\
& \quad=0 \text {. }
\end{aligned}
$$

The gauge potential is assumed to have $A_{0}$ as the only non-zero component. With its explicit spherically symmetric form

$A_{0}=K / r$

and the identity $s=2 M E$, the above equation reduces to

$$
\begin{aligned}
& \frac{d^{2} \Phi^{\prime}}{d r^{2}}-\left[\frac{\bar{l}(\bar{l}+1)}{r^{2}}-E^{2}-\frac{2\left(G_{4} s-e K\right) E}{r}\right] \Phi^{\prime} \\
& \quad=0 .
\end{aligned}
$$

It is straightforward to obtain the phase shift from here and the answer is [13]

$\delta_{\bar{l}}=\arg \Gamma\left(\bar{l}+1-i G_{4} s\right)$.

Expanding the rhs for $\bar{l} \gg 1$, we get [14]

$\delta_{\bar{l}}=-\left(G_{4} s-e K\right) \ln \bar{l}$.

The above phase shift resembles Rutherford scattering with the fine structure constant $\alpha$ being replaced by the effective coupling constant $-\left(G_{4} s-\right.$ $e K)$, which is attractive for large $s$. The phase shift can be substituted in

$f(s, t)=\frac{1}{2 i \sqrt{s}} \sum_{\bar{l}=0}^{\infty}(2 \bar{l}+1)\left[e^{2 i \delta_{\bar{l}}}-1\right] P_{\bar{l}}(\cos \theta)$ to obtain the scattering amplitude. Using the asymptotic formula for large $\bar{l}$

$P_{\bar{l}}(\cos \theta) \rightarrow J_{0}((2 \bar{l}+1) \sin \theta / 2)$,

and converting the sum into an integral as in [1], we get

$f(s, t)=-i \sqrt{s} \int_{0}^{\infty} d y y^{1-2 i G s} J_{0}\left(2 y \sqrt{s} \sin \frac{\theta}{2}\right)$,

where $y \equiv \bar{l} / \sqrt{s}$ and $t=4 E^{2} \sin ^{2} \theta / 2$. Finally, one gets

$$
\begin{aligned}
f(s, t)= & \frac{-i\left(G_{4} S-e K\right)}{\pi t} \frac{\Gamma\left(1-i\left(G_{4} s-e K\right)\right)}{\Gamma\left(1+i\left(G_{4} s-e K\right)\right)} \\
& \times\left(\frac{4}{-t}\right)^{-i\left(G_{4} s-e K\right)}
\end{aligned}
$$

The cross section follows:

$\sigma(s, t)=\frac{4\left(G_{4} s-e K\right)}{t^{2}}$.

The amplitude (10) exhibits the infinite set of 't Hooft poles at the values $G_{4} s-e K=-i N$, where $N=1,2, \ldots, \infty$. Note that for ordinary particles, $e K$ $\ll 1$ and the electromagnetic contribution to the scattering is suppressed at Planckian energies. In other words, gravity assumes the role of the dominant interaction at the Planck scale.

The linearizations of the functions $\lambda$ and $R$ are valid for spherically symmetric metrics of general relativity as well as black holes carrying RamondRamond charges. Examples in four dimensions are [15]

$d s^{2}=-f^{-1 / 2} h d t^{2}+f^{1 / 2}\left(h^{-1} d r^{2}+r^{2} d \Omega^{2}\right)$,

where $h=1-r_{0} / r, f=\prod_{i=1}^{4}\left(1+r_{i} / r\right)$ and the horizon is at $r_{0}$. This black hole metric arises when three distinct five branes of $M$-theory intersect along a line which is then wrapped on a circle. The parameters $r_{i}, i=1, . ., 4$ are related to the four $U(1)$ charges carried by the black hole, three of which are proportional to the number of the three different 5-branes, while the fourth is proportional to the Kaluza-Klein momentum along the intersection line. The ADM mass of this black hole is $M=\left(\sum_{i=1}^{4} r_{i}+2 r_{0}\right) / 4 G_{4}$. For solutions obtained by wrapping BPS saturating 
fundamental strings or R-R charged $p$-branes, the general solution is of the form [16]

$d s^{2}=-f_{p}^{-1 / 2} d t^{2}+f_{p}^{1 / 2}\left(d r^{2}+r^{2} d \Omega^{2}\right)$,

where $f_{p}=1+c_{p} / r, c_{p}$ is a constant related to the mass of the $p$-brane. The horizon is at $r=0$, and thus is singular in nature. But this metric is well behaved at large distances and gives the exact scattering amplitude as shown in the above calculation.

On the other hand, certain black holes carrying NS-NS charges have non-extremal metric of the form [17]

$$
\begin{aligned}
d s^{2}= & -\left(1-\frac{2 G M}{r}\right) d t^{2}+\frac{d r^{2}}{\left(1-\frac{2 G M}{r}\right)} \\
& +r^{2}\left(1-\frac{\alpha}{M r}\right) d \Omega^{2},
\end{aligned}
$$

where $\alpha \equiv Q^{2} e^{-2 \phi_{0}}, Q$ being the electric charge and $\phi_{0}$ the asymptotic value of the dilaton field. There is a curvature singularity at the horizon $r=\alpha / M$, which expands without limit for vanishing masses. It can be seen that the corresponding $R(r)$ (Eq. (1)) here cannot be linearised in the asymptotic $(r \rightarrow \infty)$ region, except in the extremal limit. Thus, the eikonal scattering can be computed with these metrics only in the extremal limit [3].

\section{Eikonal scattering at small string coupling}

In this section, we will compute the eikonal phase shift at weak string coupling, when the D-brane picture is appropriate. We develop a general formalism for the scattering of wrapped D-branes before specialising to four dimensions.

Consider a D- $p$-brane moving with a relative velocity $v$ with respect to a $\mathrm{D}$ - $l$-brane in 10 space-time dimensions. They are separated by a large transverse distance $b$. We assume $l \leq p$ and that none of the coordinate directions of the D-l-brane are orthogonal to those of the D-p-brane. Apart from the direction of velocity and the time coordinate, the end points of an open string ending on the two branes satisfy either Neumann (N) or Dirichlet (D) boundary conditions. We denote as $N N$ the number of string coordinates which satisfy $N$ condition at both the ends.
Similarly $N D$ and $D D$. Evidently, $D D=8-p, N N$ $=l$ and $N D=p-l$. The scattering phase shift between these two branes are given by the one loop vacuum superstring amplitude $[7,8]$

$\delta(b)=\frac{1}{2} \int_{0}^{\infty} \frac{d^{N N} k}{(2 \pi)^{(N N)}} \sum_{i} \int_{0}^{\infty} \frac{d t}{t} e^{-2 \pi \alpha^{\prime} t\left(k^{2}+M_{i}^{2}\right)}$,

where

$M_{i}^{2}=\frac{b^{2}}{4 \pi^{2} \alpha^{\prime}}+\frac{1}{\alpha^{\prime}} \sum$ (oscillators)

The oscillator sum and the integral finally yields

$$
\begin{aligned}
\delta(b)= & \frac{1}{4 \pi} \int_{0}^{\infty} \frac{d t}{t}\left(8 \pi^{2} \alpha^{\prime} t\right)^{-N N / 2} e^{-b^{2} t / 2 \pi \alpha^{\prime}} \\
& \cdot(B \times J),
\end{aligned}
$$

where $B, J$ are the bosonic and fermionic contributions to the oscillator sum in the in the one loop open superstring amplitude given by [8]

$$
\begin{aligned}
B= & f_{1}^{-(N N+D D)}(q) f_{4}^{-N D}(q) \frac{\Theta_{1}^{\prime}(0, i t)}{\Theta_{1}(\epsilon t, i t)}, \\
J= & \frac{1}{2}\left[-f_{2}^{N N+D D}(q) f_{3}^{N D}(q) \frac{\Theta_{2}(\epsilon t, i t)}{\Theta_{2}(0, i t)}\right. \\
& +f_{3}^{N N+D D}(q) f_{2}^{N D}(q) \frac{\Theta_{3}(\epsilon t, i t)}{\Theta_{3}(0, i t)} \\
& \left.-f_{4}^{8}(q) \frac{\Theta_{4}(\epsilon t, i t)}{\Theta_{4}(0, i t)} \delta_{p, l}\right],
\end{aligned}
$$

where $q \equiv e^{-\pi t}$,

$f_{1}(q)=q^{1 / 12} \prod_{n=0}^{\infty}\left(1-q^{n}\right)$,

$f_{2}(q)=\sqrt{2} q^{1 / 12} \prod_{n=0}^{\infty}\left(1+q^{n}\right)$

$f_{3}(q)=q^{-1 / 24} \prod_{n=0}^{\infty}\left(1+q^{2 n-1}\right)$,

$f_{4}(q)=q^{-1 / 24} \prod_{n=0}^{\infty}\left(1-q^{2 n-1}\right)$.

The rapidity $\epsilon$ is defined as $\tanh \pi \epsilon=v$. Note that the last term in $J$ comes from summation of the $N S(-1)^{F}$ sector and contributes only when $N D=0$. 
This term is due to R-R exchange, and we will concentrate on this term in the next section when we look at charged particle amplitudes.

Now, to compare the D-brane results with the results on the black hole side, we have to compactify the branes on suitable compact manifolds, such that in the non-compact space-time they look like point particles. For simplicity, we compactify on a $c$ dimensional torus (with $p \leq c$ ), such that the resultant noncompact space time is a $(10-c)$ dimensional with a Lorentzian signature. We take the range of each coordinate of the torus to be $L_{i}, i=1, \ldots, c$. Thus, the volume of the torus is $V=\prod L_{i}$. To obtain the phase shift for these wrapped D-branes, the formula (16) has to be modified. For each compactified $N N$ direction, the momentum integral is replaced by a discrete sum in the one loop amplitude. In other words the momentum integral is restricted to the remaining non-compact $N N$ directions, and and a factor of $\Theta_{3}\left(0,8 \pi^{2} \alpha^{\prime} t / L^{2}\right)$ is inserted in the integrand for each compactified direction. Similarly, for each compact $D D$ direction, a sum over winding modes is introduced, resulting in a factor of $\Theta_{3}\left(0, i t L^{2} / 2 \pi^{2} \alpha^{\prime}\right)$ in the integrand [9]. Since all the $N N=l$ coordinates are compactified and there are $c-p$ compact $D D$ coordinates, the final result is

$$
\begin{aligned}
\delta(b)= & \frac{1}{4 \pi} \int_{0}^{\infty} \frac{d t}{t} e^{-b^{2} t / 2 \pi \alpha^{\prime}} \\
& \times\left[\prod_{i=1}^{l} \Theta_{3}\left(0,8 i \pi^{2} \alpha^{\prime} t / L_{i}^{2}\right)\right] \\
& \times\left[\prod_{i=p+1}^{c} \Theta_{3}\left(0, i t L_{i}^{2} / 2 \pi \alpha^{\prime}\right)\right] \cdot(B \times J) .
\end{aligned}
$$

Now, large impact parameter $(b \rightarrow \infty)$, scattering is dominated by the exchange of massless closed string states, for which it is sufficient to restrict the integrand in the regime $t \rightarrow 0[10,18]$. Using the relevant formulae given in $[9,19]$, we get

$$
\begin{aligned}
& \Theta_{3}\left(0,8 i \pi^{2} \alpha^{\prime} t / L\right) \rightarrow \frac{L}{2^{3 / 2} \pi \sqrt{\alpha^{\prime}}} \frac{1}{\sqrt{t}}, \\
& \Theta_{3}\left(0, i t L^{2} / 2 \pi^{2} \alpha^{\prime}\right) \rightarrow \frac{2^{1 / 2} \pi \sqrt{\alpha^{\prime}}}{L} \frac{1}{\sqrt{t}},
\end{aligned}
$$

$$
\begin{aligned}
B \rightarrow & 2^{-(p-l) / 2} \pi t^{[3-(p-l) / 2]} e^{2 \pi / 3 t} \frac{e^{-\pi \epsilon^{2} t}}{\sinh \pi \epsilon}, \\
J \rightarrow & 4 e^{-2 \pi / 3 t} e^{\pi \epsilon^{2} t} \\
& \times\left[2-(p-l) / 2+\sinh ^{2} \pi \epsilon-2 \delta_{p, l} \cosh \pi \epsilon\right],
\end{aligned}
$$

and the phase shift becomes

$\delta(b)=\Lambda \kappa(\epsilon) \int_{0}^{\infty} \frac{d t}{t} e^{-b^{2} t / 2 \pi \alpha^{\prime}} \frac{t^{3}}{t^{c / 2}}$,

where

$$
\begin{aligned}
& \Lambda=\frac{2^{c / 2} \pi^{c-(p+l)} \sqrt{\alpha^{\prime}}{ }^{c-(p+l)}}{2^{p+l}}\left(\frac{\prod_{i=1}^{l} L_{i}}{\prod_{j=p+1}^{c} L_{j}}\right), \quad \text { (29) } \\
& \kappa(\epsilon)=\frac{2-(p-l) / 2+\sinh ^{2} \pi \epsilon-2 \delta_{p, l} \cosh \pi \epsilon}{\sinh \pi \epsilon} .
\end{aligned}
$$

Note that the integral in the above expression is independent of $p$ and $l$ and depends only on $c$. That is, it is the same for branes of arbitrary dimensions for a given compactification. Thus, scattering phase shifts for all $p, l$ can be calculated from the above expression provided the branes completely wrap on the internal torus. Also, as expected, $K(\epsilon=0)=0$ for $p=l$ or $p-l=4$. This is the familiar no-force condition for BPS states.

However, for our present purposes, we specialise to the case of $c=6$, i.e. scattering in 4 dimensional non-compact space time. Then the integral over $t$ in (28) simply yields a factor $-2 \ln \left(b / \sqrt{2 \pi \alpha^{\prime}}\right)$. In addition, we make the ultrarelativistic approximation $v \rightarrow 1, \epsilon \rightarrow \infty$, such that

$\kappa(\epsilon) \rightarrow \frac{1}{2} e^{\pi \epsilon}$.

Then Eq. (28) becomes

$$
\delta(b)=-\frac{\prod_{i=1}^{l} L_{i} \prod_{j=1}^{p} L_{j} e^{\pi \epsilon}}{2^{p+l-3} \pi^{p+l-6}{\sqrt{\alpha^{\prime}}}^{p+l-6} V} \ln \frac{b}{\sqrt{2 \pi \alpha^{\prime}}} .
$$


Rewriting this amplitude in terms of the masses of the branes given by

$m_{p(l)}=\frac{\prod_{i=1}^{p(l)} L_{i}}{g(2 \pi)^{p(l)} \sqrt{\alpha^{\prime}} p(l)+1}$,

and the Newton's constant in $(10-c)$ dimensions (for $c=6$ )

$G_{10-c}=\frac{8 \pi^{6} g^{2} \alpha^{\prime 4}}{V}$

yields

$\delta(b)=-G_{4} m_{p} m_{l} e^{\pi \epsilon} \ln \frac{b}{\sqrt{2 \pi \alpha^{\prime}}}$.

Finally, using $s=m_{p} m_{l} \exp (\pi \epsilon)$, which simply expresses the relativistic transformation of energy, and $\bar{l}=b E$, the amplitude takes the form

$$
\delta(b)=-G_{4} \operatorname{s} \ln \frac{\bar{l}}{E \sqrt{2 \pi \alpha^{\prime}}},
$$

(the factor $E \sqrt{2 \pi \alpha^{\prime}}$ is irrelevant as it does not appear in the scattering amplitude and the cross section, and will simply be dropped).

Thus not only is the phase shift exactly calculable in the weak coupling regime, comparison with (7) (for $e=0$ ) shows that it perfectly agrees with that calculated in the strong coupling regime, implying that there is no discontinuity in the point particle scattering amplitude as one tunes the string coupling, for arbitrary masses of particles. Moreover, as expected, the gravitational interaction dominates overwhelmingly over gauge interactions.

\section{Inclusion of charge}

To extend the results of the previous section to include gauge interactions, the charge interaction term proportional to $\cosh \pi \epsilon$ has to be retained in the kinematical factor (30). This vanishes unless $p=l$, since branes of different dimensions do not couple to each other via gauge fields. Thus for $p=l$

$\kappa(\epsilon) \rightarrow \frac{1}{2}\left(e^{\pi \epsilon}-4\right)$.
As before, introducing $m_{p}$ and $G_{4}$ gives

$$
\begin{gathered}
\delta(b)=-G_{4} m_{p}^{2}\left(e^{\pi \epsilon}-4\right) \ln \bar{l} \\
=-\left(G_{4} s-4 G_{4} m_{p}^{2}\right) \ln \bar{l} .
\end{gathered}
$$

Let us now consider the phase shift obtained using the black hole background, Eq. (7). Here, inclusion of charge shows that the coupling constant is given by $G s-e K$. To determine $K$, we look at the $p+1$ form potentials due to the static brane to which the relativistic $p$ brane couples. In 10-dimensions the asymptotic value of the $p+1$ potential is given by [10]

$A_{p+1}=\frac{q_{p}}{r^{7-p}} d t \wedge d x \wedge \ldots \wedge d x^{p}$,

where

$q_{p}=2^{5-p} g \pi^{\frac{5-p}{2}}{\sqrt{\alpha^{\prime}}}^{7-p} \Gamma\left(\frac{7-p}{2}\right)$.

The effective $U(1)$ potential due to the brane living in $R^{10-c} \times T^{c}$ is obtained in two steps: Firstly, a Kaluza Klein reduction is performed on the 10-dimensional $p+1$ potential to obtain a one form potential in $10-p$ dimensions due to the brane completely wrapped on $T^{p}$ [20]:

$A_{1}=\frac{q_{p}}{r^{7-p}} d t$.

Secondly, for the remaining $c-p$ compact directions, which are transverse to the brane vertical reduction is performed. For this, we stack the Kaluza-Klein reduced configurations in the compact directions transverse to the brane and go to the continuum limit by integrating over the latter, resulting in one form potentials in $10-c$ dimensions [20]

$$
\begin{aligned}
& A_{1}=\frac{q_{p} \prod_{i=1}^{p} L_{i}}{V} \int_{o}^{\infty} \frac{d^{c-p} r_{\perp}}{\left[r^{2}+r_{\perp}^{2}\right]^{\frac{7-p}{2}}} d t \\
& =\frac{q_{p} \prod_{i=1}^{p} L_{i}}{V} \int d \Omega_{c-p-1} \int_{0}^{\infty} \frac{r_{\perp}^{c-p-1} d r}{\left[r^{2}+r_{\perp}^{2}\right]^{\frac{7-p}{2}}} d t
\end{aligned}
$$


where $r_{\perp}$ refers to the transverse distance from the $p$-brane, and $\Omega_{c-p-1}$ is the volume of the unit $(c-p-1)$ sphere, given by

$\Omega_{c-p-1}=\frac{2 \pi^{\frac{c-p}{2}}}{\Gamma\left(\frac{c-p}{2}\right)}$.

The integral is elementary, and when expressed in terms of the $p$-brane mass and the Newton's constant given Eqs. (32) and (33) respectively, we get

$A_{1}=\frac{4 G_{10-c} \Gamma\left(\frac{7-c}{2}\right)}{\pi^{\frac{7-c}{2}} r^{7-c}} m_{p} d t$.

Comparing with the expression (4), we conclude that

$K=\frac{4 G_{10-c} \Gamma\left(\frac{7-c}{2}\right)}{\pi^{\frac{7-c}{2}}} m_{p}$.

Now, the charge of the moving brane is $e=m_{p}$, by the BPS condition. Consequently, for $c=6$, the scattering phase shift (7) will be modified as

$\delta(b)=-\left(G_{4} s-4 G_{4} m_{p}^{2}\right) \ln \bar{l}$

which precisely agrees with the phase shift (37) obtained in the D-brane side.

\section{Discussions}

We have shown that the eikonal scattering amplitude obtained by modelling the point particles as black holes is exactly reproduced by eikonal scattering of wrapped D-branes. In this regime only the gravitational field at infinity is probed (as the black hole metric is linearised) and the details of the metric are not realised. So, one should study the corrections to the eikonal phase shift, as the impact parameter and velocity are tuned to smaller values, and see whether the details begin to emerge [12,21]. Our results are independent of the dimensions of the brane but the kinematical factor $\kappa(\epsilon)$, and hence $\delta(b)$ ceases to be independent of $p$ and $l$ once one relaxes the condition $\epsilon \rightarrow \infty$.
We know that the type II B string theory has S-duality group as SL(2,Z) which relates fundamental strings to the D-strings. Under this S-duality operation, gravitons are left invariant and NS-NS charged fields become R-R charged fields. Our Dbrane calculation for gravitational exchange (dominant term) matches the leading order term (eikonal limit) in the scattering amplitude for fundamental strings [22]. This confirms the S-duality symmetry. Moreover, we have obtained the subdominant term (eikonal limit) due to R-R charged field exchange between the two D-p-branes. Invoking the S-duality, we can say that this must be also be the amplitude for NS-NS gauge field exchange in fundamental string scattering. In [22], corrections to the eikonal fundamental string-string graviton exchange amplitude were calculated and shown to be order $1 / \bar{l}^{2}$. It will be interesting to see whether the D-brane scattering amplitude gives the same.

Though the fundamental string and the D-brane scattering amplitudes are same, the relevant energy scales for eikonal scattering are different for the two. For the D-p-branes to be relativistic, the condition

$E \gg m_{p}$

must be imposed on their energy. Using the expression for the brane mass, we get

$E \gg \frac{L^{p}}{g{\sqrt{\alpha^{\prime}}}^{p+1}}$.

As is evident, in 10-dimensions, the mass of the D-0 brane is much larger than the Planck mass $M_{\mathrm{pl}}$ and the string mass $m_{s} \sim 1 / \sqrt{\alpha}$. Hence for condition (45) to be realised, energies relevant for D-0 brane eikonal scattering has to be much larger than both $M_{\mathrm{pl}}$ and $m_{s}$. In other words for D-0 branes to become relativistic, we need to consider regimes where $s g^{2} \alpha^{\prime} \gg 1$, (In the c.m. frame $s=E^{2}$ ). (Note that for non-perturbative effects of M-theory to become important, we need to have $\operatorname{tg}^{2} \alpha^{\prime} \rightarrow 1$ [18], and we are not probing that regime.) On the other hand, for fundamental strings, $s \alpha^{\prime} \gg 1$ gives the eikonal limit, as considered in [22]. Following [23] we take $1 / g \sqrt{\alpha}$ as the energy scale in our problem. and the condition on the compactification volume, from Eq. (44) is

$V_{p} \ll \sqrt{\alpha}^{\prime}$, 
which implies that the compactification radii should be sufficiently small compared to the string scale. Note that if we had used $1 / \sqrt{\alpha}$, as in [22] the conditions on compactification lengths would have become $g$ dependent, and difficult to interpret.

Another interesting observation is that though the D-brane scattering amplitude includes all long range closed string exchanges, only the graviton exchange dominates in the above kinematical regime ${ }^{5}$. This was anticipated in the black hole calculation by 't Hooft and the weak coupling calculation vindicates this. Our weak coupling calculations can be generalised to higher dimensions. For example, in five non-compact dimensions, it is easy to see that the phase shift goes as $1 / b$, which is the Green's function for three transverse dimensions.

One can try to examine more sophisticated compactifications e.g. on $K 3$ to see whether similar conclusions hold for those situations also [25]. We hope to report on it in the near future.

\section{Acknowledgements}

S.D. would like to thank C. Bachas, F. Hussain, R. Iengo and K. S. Narain for useful discussions and the International Centre for Theoretical Physics, Trieste for hospitality where part of this work was done. A.D. would like to thank Theoretical Physics Group, Tata Institute of Fundamental Research, Mumbai for hospitality during the course of this work and P. Majumdar for useful discussions.

\section{References}

[1] E. Verlinde, H. Verlinde, Nucl. Phys. B 371 (1992) 246, hep-th/9110017.

[2] T. Dray, G. 't Hooft, Nucl. Phys. B 253 (1985) 173; G. 't Hooft, Phys. Lett. B 253; S. Das, P. Majumdar, Phys. Rev. D 51 (1995) 5664.

\footnotetext{
${ }^{5}$ This was also observed in [24]
}

[3] S. Das, P. Majumdar, Phys. Rev. D 55 (1997) 2090.

[4] A. Strominger, C. Vafa, Phys. Lett. B 379 (1996) 99, hepth/9601029; G. Horowitz, Phys. Rev. D 55 (1997) 835, gr-qc/9604051, and references therein.

[5] C. Callan, J.M. Maldacena, Nucl. Phys. B 472 (1996) 591, hep-th/9602043; J.M. Maldacena, L. Susskind, Nucl. Phys. B 475 (1996) 679, hep-th/9604042; A. Dhar, G. Mandal, S.R. Wadia, Phys. Lett. B 388 (1996) 51, hep-th/9605234; S.R. Das, S.D. Mathur. Nucl. Phys. B 478 (1996) 561, hep-th/9606185.

[6] G.T. Horowitz, J. Polchinski, A Correspondence Principle for Black Holes and Strings, hep-th/9612146.

[7] C. Bachas, Phys. Lett. B 374 (1996) 37, hep-th/9511043.

[8] G. Lifschytz, Phys. Lett. B 388 (1996) 720, hep-th/9604156.

[9] G. Lifschytz, Nucl. Phys. B 499 (1997) 283, hep-th/9610125.

[10] V. Balasubramanian, F. Larsen, Nucl. Phys. B 506 (1997) 61, hep-th/9703039.

[11] B.P. Mandal, S. Mukhopadhyay, D-brane interaction in the type IIB Matrix Model, hep-th/9709098.

[12] I.V. Volovich, Planckian Energy Scattering of D-branes and M(atrix) Theory in Curved Space, hep-th/9705013.

[13] L.D. Landau, E.M. Lifschitz, Quantum Mechanics, Pergamon Press, 1959, Section 36.

[14] M. Abramowitz, I.A. Stegun (Eds.), Handbook of Mathematical Functions, Dover Publications, Inc., New York, 1965.

[15] S.S. Gubser, I.R. Klebanov, Phys. Rev. Lett. 77 (1996) 4491, hep-th/9609076, and references therein.

[16] J.M. Maldacena, Black Holes in String Theory, Ph.D. Thesis, Princeton University, June 1996, hep-th/9607235.

[17] D. Garfinkle, G. Horowitz, A. Strominger Phys. Rev. D 43 (1991) 3140; D 45 (1992) 3888 (E).

[18] M. Douglas, D. Kabat, P. Pouliot, S. Shenker, Nucl. Phys. B 485 (1997) 85, hep-th/9608024.

[19] A. Erdélyi (Ed.), Higer Transcendental Functions (Bateman Manuscript Project), vol. 2, McGraw-Hill Book Co., Inc., New York, 1953, pp. 355-371.

[20] H. Lu, C.N. Pope, K.S. Stelle, Nucl. Phys. B 481 (1996) 313, hep-th/9605082; K.S. Stelle, Lectures on Supergravity pbranes, hep-th/9701088.

[21] D. Amati, M. Ciafaloni, G. Veneziano, Nucl. Phys. B 47 (1990) 550; S. Das, P. Majumdar, hep-th/9504060.

[22] D. Amati, M. Ciafaloni, G. Veneziano, Phys. Lett. B 197 (1987) 81; Int. J. Mod. Phys. A 3 (1988) 1615.

[23] S. Shenker, Another Length Scale in String Theory?, hepth/9509132.

[24] F. Hussain, R.I.C. Núñez, C.A. Scrucca, Phys. Lett. B 409 (1997) 101, hep-th/9706186.

[25] M. Douglas, H. Ooguri, S. Shenker, Phys. Lett. B 402 (1997) 36, hep-th/9702203. 